\title{
Practical Aspects for a Working Compile Time Garbage Collection System for Mercury
}

\author{
Nancy Mazur ${ }^{1}$, Peter Ross ${ }^{2}$, Gerda Janssens ${ }^{1}$, and Maurice Bruynooghe ${ }^{1}$ \\ 1 Department of Computer Science, K.U.Leuven \\ Celestijnenlaan, 200A, B-3001 Heverlee, Belgium \\ \{nancy, gerda, maurice\}@cs.kuleuven.ac.be \\ 2 Mission Critical, Drève Richelle, 161, Bât. N \\ B-1410 Waterloo, Belgium \\ petdr@miscrit.be
}

\begin{abstract}
Compile-time garbage collection (CTGC) is still a very uncommon feature within compilers. In previous work we have developed a compile-time structure reuse system for Mercury, a logic programming language. This system indicates which datastructures can safely be reused at run-time. As preliminary experiments were promising, we have continued this work and have now a working and well performing near-toship CTGC-system built into the Melbourne Mercury Compiler (MMC). In this paper we present the multiple design decisions leading to this system, we report the results of using CTGC for a set of benchmarks, including a real-world program, and finally we discuss further possible improvements. Benchmarks show substantial memory savings and a noticeable reduction in execution time.
\end{abstract}

\section{Introduction}

Modern programming languages typically limit the possibilities of the programmer to manage memory directly. In such cases allocation and deallocation is delegated to the run-time system and its garbage collector, at the expense of possible run-time overhead. Declarative languages go even further by prohibiting destructive updates. This increases the run-time overhead considerably: new datastructures are created instead of updating existing ones, hence garbage collection will be needed more often.

Special techniques have been developed to overcome this handicap and to improve the memory usage, both for logic programming languages $[10,14,18]$ and functional languages $[21,16]$. Some of the approaches depend on a combination of special language constructs and analyses using unique objects $[19,1,22]$, some are solely based on compiler analyses $[13,16]$, and others combine it with special memory layout techniques [21]. In this work we develop a purely analysis based memory management system.

Mercury, a modern logic programming language with declarations [19] profiles itself as a general purpose programming language for large industrial projects. Memory requirements are therefore high. Hence we believe it is a useful research 
goal to develop a CTGC-system for this language. In addition, mastering it for Mercury should be a useful stepping stone for systems such as Ciao Prolog [12] (which has optional declarations and includes the impurities of Prolog) and HAL [8] (a Mercury-based constraint language).

The intention of the CTGC-system is to discover at compile-time when data is not referenced anymore, and how it can best be reused. Mulkers et al. [18] have developed an analysis for Prolog which detects when memory cells become available for reuse. This analysis was first adapted to languages with declarations [3] and then refined for use in the presence of program modules [15]. A first prototype implementation was made to measure the potential of the analysis for detecting dead memory cells. As the results of the prototype were promising [15], we have continued this work and implemented a full CTGC-system for the Melbourne Mercury Compiler (MMC), focusing on minimizing the memory usage of a program. In this paper we present the different design decisions that had to be taken to obtain noticeable memory savings, while remaining easy to implement within the MMC and with acceptable compilation overhead. A series of benchmarks are given, measuring not only the global effect of CTGC, but also the effect of the different decisions during the CTGC analysis.

After presenting some background in Section 2, we first solve the problem of deciding how to perform reuse once it is known which cells might die (Section 3). Section 4 presents low-level additions required to increase precision and speed, and obtain the first acceptable results for a set of benchmarks (Section 5). Using cell-caching (Section 6) more memory savings can be obtained. Finally improvements related to other work are suggested (Section 7 ), followed by a conclusion (Section 8).

\section{Background}

\subsection{Mercury}

Mercury [11] is a logic programming language with types, modes and determinism declarations. Its type system is based on a polymorphic many-sorted logic and its mode-system does not allow partially instantiated datastructures.

The analysis performed by our CTGC-system is at the level of the High Level Data Structure (HLDS) constructed by the MMC. Within this structure, predicates are normalized, i.e. all atoms appearing in the program have distinct variables as arguments, and all unifications $X=Y$ are explicited as one of (1) a test $X==Y$ (both are ground terms), (2) an assignment $X:=Y,(X$ is free, $Y$ is ground) (3) a construction $X \Leftarrow f\left(Y_{1}, \ldots, Y_{n}\right)$ ( $X$ is free, all $Y_{i}$ are ground), or (4) a deconstruction $X \Rightarrow f\left(Y_{1}, \ldots, Y_{n}\right)$ ( $X$ is ground, all $Y_{i}$ are free) [11]. Within the HLDS, the atoms of a clause body are ordered such that the body is well moded. In the paper, we will use the explicit modes.

Just like in the HLDS we will use the notion of a procedure, i.e. a combination of one predicate with one mode, and thus talk about the analysis of a procedure. 


\subsection{General Structure of the CTGC-System}

The CTGC-system consists of a data-flow analysis, followed by a reuse analysis and ended by a code generation pass (similar to [10]).

The data-flow analysis is performed to obtain structure-sharing information (expressed as possible aliases [3]) and to detect when heap cells become dead and are therefore available for reuse. It is based on abstract interpretation [2] using a so called default call pattern for each of the procedures to be analysed. This default call pattern makes minimal realistic assumptions: the inputs to a procedure are in no way aliased, and only the outputs will be used after the call to the procedure. The data-flow analysis requires a fixpoint computation to deal with recursive predicate definitions. For more details, see [3,15].

Next the reuse analysis decides which reuses are possible (see Section 2.5). Different versions can then be created for the different reuses detected. While the underlying concepts were already developed in [15], the pragmatics of our implementation are discussed in this paper.

Finally, low-level code corresponding to the detected reuses is generated.

As Mercury allows programming with modules, the CTGC-system processes each module independently. Interface files are used to allow analysis information (structure-sharing and reuse information) generated while processing one module to be used when processing other modules.

\subsection{Data Representation}

The purpose of the CTGC-system is to identify which objects on the heap, so called datastructures, become dead and can therefore be reused. In order to understand what these objects are, we will clarify the way typed terms are usually $^{1}$ represented in the MMC. Consider the following types:

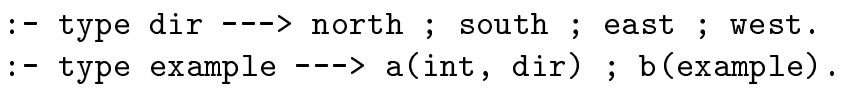

Terms of primitive types such as integers, chars, floats ${ }^{2}$ and pointers to strings are represented as single machine words. Terms of types such as dir, in which every alternative is a constant are equivalent to enumerated types in other languages. Mercury represents them as consecutive integers starting from zero, and stores them in a single machine word. Terms of types such as example are stored on the heap. The pointer to the actual term on the heap is tagged [9]. This tag is used to indicate the function symbol of the term. Terms of types having more function symbols than a single tag can distinguish use secondary tags.

Figure 1 shows the representation of a variable A bound to $b(a$ ( 3 , east)). In this paper ha1, hy $1, \ldots$ denote heap cells, whereas $\mathbf{s a}, \mathbf{s x}, \ldots$ are registers or stack locations.

\footnotetext{
${ }^{1}$ The MMC compiles to different back-ends, the most common being ANSI-C. Higherlevel back-ends, such as Java or .NET, use different low level representations, yet the theory of recycling heap cells remains the same.

${ }^{2}$ Depending on the word-size, these might have a boxed representation.
} 


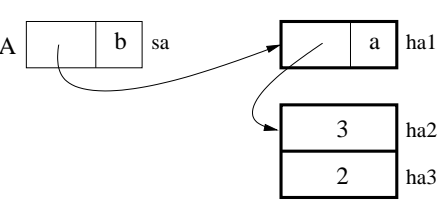

Fig. 1. $A=b(a(3$, east $))$.

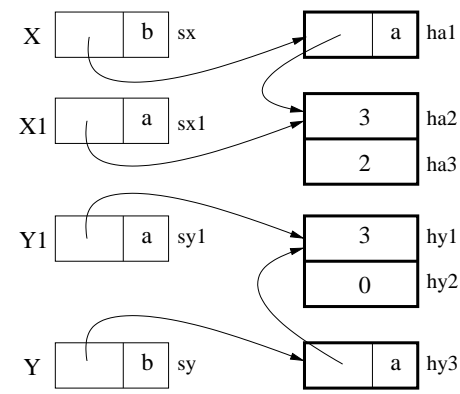

Fig. 3. No reuse

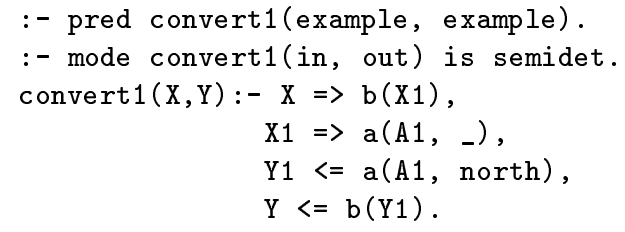

Fig. 2. Conversion-procedure.

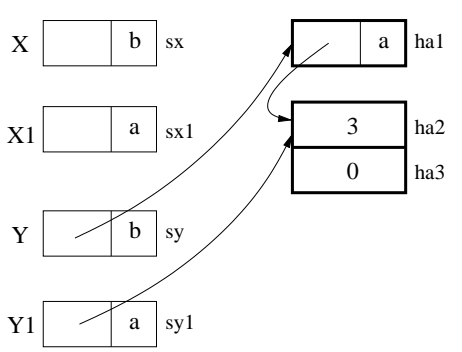

Fig. 4. Reuse

\subsection{Data Reuse}

Figure 3 shows the memory layout when calling convert1(A, B) (Fig. 2), where $A$ is bound to $b$ (a (3, east) (Fig. 1).

After deconstructing the input, new heap cells (hy1, hy2 and hy3) are allocated to create $\mathrm{Y}$, and the content of $\mathrm{X}$ is partially copied into those cells. If it can be shown at compile-time that after this procedure call the term pointed at by $\mathrm{X}$ will not be referenced during the rest of the program (thus becoming available for reuse), then the deconstruction statements perform the last access ever to the concerned heap cells (ha1, ha2, ha3) after which they become garbage, and can be (re)used for Y (Fig. 4, the contents of sx and sx1 are no longer relevant).

The optimization could go further and detect that this reuse only requires the update of one heap cell, namely ha3. Yet currently we mainly focus on the memory usage of a program, execution time being only of indirect importance. Therefore we do not try to optimize the number of field updates in the presence of reuse. See also Section 7.

\subsection{Types of Reuse}

During reuse analysis we make a distinction between different kinds of reuses [15]. The procedure shown in Fig. 2 has direct reuse (of $\mathrm{x}$ ): it might contain the last reference to the cells of $\mathrm{X}$ which can then be reused by $\mathrm{Y}$. However, the reuse is 
conditional: if the caller's environment does not correspond to the default call pattern (e.g. keeping a reference to one of the cells marked for reuse), reuse is not allowed. As this imposes very harsh restrictions on the reuse possibilities, we introduced the notion of reuse conditions which express the minimal conditions a call pattern has to meet so that reuse is safe. These conditions are expressed in terms of the variables involved (here $\mathrm{X}$ ). If the reuse is independent of the calling environment ( $\mathrm{X}$ being a local variable), then we have unconditional reuse.

Given the reuse conditions, the next step of the reuse analysis is to check for indirect reuses. Consider the following procedure:

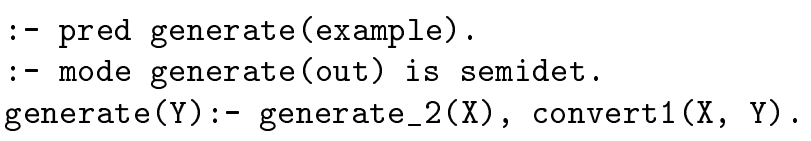

Assuming the default call pattern for generate, the call to convert1 meets the condition that after that call $\mathrm{X}$ will not be used anymore. Hence, a reuse-version of convert1 can be called and we say that generate has indirect reuse. Moreover, $\mathrm{X}$ is a local variable, so reusing it will always be safe as it is independent of the call pattern to generate. This is an example of unconditional (indirect) reuse. If $\mathrm{X}$ would have been an input variable to generate, the indirect reuse would be conditional and additional reuse conditions would be formulated.

\section{A Working Reuse Decision Approach}

Consider the predicate in Fig. 5 which converts a list of data-elements into a new list of data-elements. While the data-flow analysis spots the datastructures that can potentially be reused, it is up to the reuse analysis to select those reuses (direct and indirect) that yield the most interesting saving w.r.t. memory usage (and indirectly execution time).

\subsection{Deciding Direct Reuse}

A first restriction we impose is to limit reuses to local reuses, i.e. a dead cell can only be reused in the same procedure as where it is last accessed (deconstructed). In Section 6 we discuss techniques of how to lift this restriction. Furthermore, we consider that dead structures can only be reused by at most one new structure. Using the terminology of Debray [7], we limit ourselves to the simple reuse problem. It is not difficult to remove this limitation, but it makes the reuse decisions more complex. We plan to lift this restriction in the future.

The data-flow analysis of the example identifies the deconstructed datastructures (at d1, resp. d2) as available for reuse. The procedure also contains two constructions ( $c 1$ and $c 2$ ) where the memory from the dead cells could be reused.

Each of the combinations yields an acceptable reuse-scheme. Yet, which one is the most interesting? It has been shown that this problem [7] can be reformulated as an instance of the maximum weight matching problem for a weighted bipartite graph. However for simplicity of implementation we have reduced this 


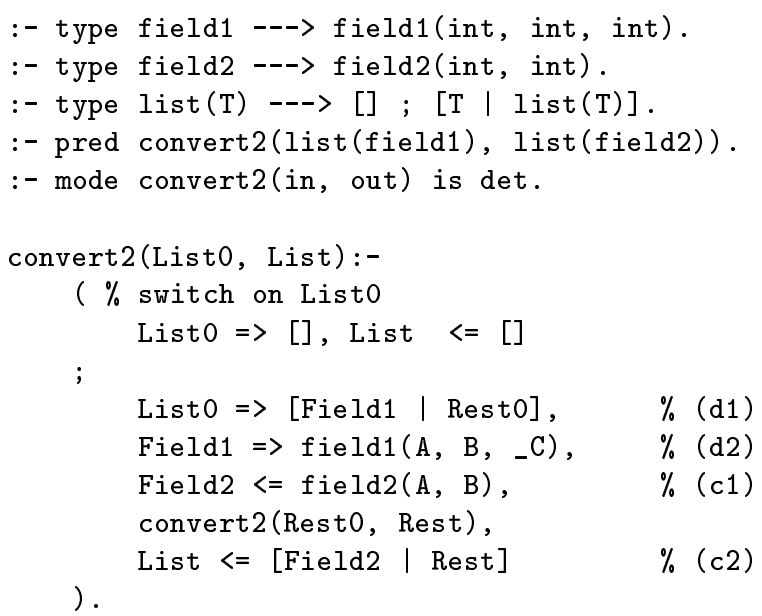

Fig. 5. Converting lists.

general matching problem to two orthogonal decisions: imposing constraints on the allowed reuses, and using simple strategies to select amongst different candidates for reuse. We will discuss each of these.

Constraints on allowed reuses. Constraints allow one to express common characteristics between the dead and the newly constructed cell and reflect the restrictions which can be imposed by the back-end to which a Mercury program is compiled.

We have implemented the following constraints:

- Almost matching arities. This constraint expresses the intuition that it can be worthwhile to reuse a dead cell, even if not all memory-words are reused. This is indeed interesting if it can be guaranteed that the superfluous words will be collected by the run-time garbage collector within a reasonable delay. In our example, allowing a difference of size one allows c1 and c2 to reuse the memory available from either $\mathrm{d} 1$ or $\mathrm{d} 2$.

- Matching arities. If the run-time system is not powerful enough to be used with almost matching arities, then a more restrictive constraint can be used: only allow reuse between constructors having the same arity. This means that in our example only d1 can be reused (by either c1 or c2).

- Label-preserving. Using the Java or .NET back-end, it is not possible to change the type of run-time objects, therefore reuse is only allowed if the dead and new cell have the same constructor (label). For the example this means that the cell from d1 can only be reused in c2.

Selection strategies. When a cell can reuse different dead cells, a choice has to be made (e.g. c1 can either reuse the cell available from d1 or d2). Some 
choices yield better results than others. We have experimented with two simple strategies:

- Lifo. Traverse the body of the procedure and assign the reuses using a lastin-first-out selection strategy. This means that when a choice is left for a given construction, choose the cell which died most recently. The intuition is that after deconstructing a variable, it is very likely that a new similar cell will be constructed in the same context.

e.g. If $\mathrm{c} 1$ is allowed to reuse the cells from $\mathrm{d} 1$ or $\mathrm{d} 2$, then according to this strategy, Field1 will be reused for constructing Field2 and Listo for List.

- Random. The intuition behind the lifo-strategy might not always be true, for example in the presence of a disjunction ${ }^{3}$. Therefore we have added a simple strategy which randomly selects the dead cell amongst all the candidates.

\subsection{Deciding Indirect Reuse}

In order to decide whether a call to a procedure can be substituted by a call to a reuse version of that procedure, we must be sure that such substitution is safe. This is tested by checking the reuse-conditions (under the assumption of a default call pattern). If it is safe to call the reuse-version we have to decide whether we will do so or not.

Here we have decided for simplicity by always calling the reuse-version of a procedure if it is safe to do so. In Section 7 we discuss the drawbacks and suggest a possible better solution.

Suppose that for our previous example we would only allow the reuse of the list-cells ( $\mathrm{d} 1$ by $\mathrm{c} 2$ ). Such reuse is conditional: the list cell only dies iff it is not needed within the caller's context. This condition has to be checked for the recursive call. Under the default call pattern (see Section 2.4) Rest0 is dead at the moment of the recursive call, hence the condition is satisfied, and the recursive call can safely be substituted by a call to its reuse version ${ }^{4}$.

\subsection{Splitting into Different Versions}

Once the possible direct and indirect reuses have been decided, there is one remaining decision left: how many versions of a given procedure should be created? In our example, we might have detected three reuses: List0 reused by List, Field1 by Field2, and the indirect reuse (the recursive call to the reuse version). We can generate 4 interesting versions of the initial procedure: a version with no reuse, a version reusing only Listo, a version reusing Field1 and a version reusing both (where the reuse versions also include the recursive reuse call). In general, for a procedure with $n$ possible direct reuses, $2^{n}$ interesting

\footnotetext{
${ }^{3}$ e.g. $X \Rightarrow f(\ldots),(\ldots Y<=f(\ldots) ; \ldots), Z<=f(\ldots)$ : as the first branch of the disjunction might not always be executed, it is more interesting to allow $\mathrm{Z}$ to reuse $\mathrm{X}$ than $\mathrm{Y}$.

${ }^{4}$ Note that this indirect reuse is in itself conditional: it can only be allowed if the list-cells of Rest0 are not needed in the callers context.
} 
versions can be created. In our implementation we limit the number of versions to at most two: a version which imposes no conditions on the caller (containing all possible unconditional reuses), and a version containing all detected reuses. In Section 7 we briefly discuss other possibilities.

\section{Low Level Additions}

Given the previous decisions, a first CTGC-system was implemented. Although good results were obtained for small programs (e.g. naive-reverse), we ran into problems when analysing large ones:

- imprecision in the alias analysis had the effect that relatively few cells were recognized as dead.

- the number of aliases collected within a procedure became huge. This slowed down the operations manipulating them and the CTGC process became too time consuming.

\subsection{Enhancing the Aliasing Precision}

The underlying analysis for deriving alias-information uses the concept of top which expresses that all data parts might be aliased. This is a safe abstraction in the case of total lack of knowledge about the possible existing aliases at some program point. Once generated, this lack of information propagates rapidly as all primitive operations manipulating it yield top as well.

Such a top is generated in the presence of language constructs with which the analysis cannot cope yet. These are procedures defined in terms of foreign code $(c, \mathrm{C}++)$, higher-order calls and typeclasses. It is also generated for procedures which are defined in other modules that have not yet been analysed and for which no interface files have been generated yet.

To obtain a usable CTGC-system, techniques were needed to limit the creation and propagation of top. In our implementation, three techniques are used:

1. Using heuristics. Based on the type- and mode- declaration of a procedure, one can derive whether it can create additional aliases or not, without looking at the procedure's body. This is the case when a procedure uses unique objects (declared di or uo [11]), or only has unique output variables ${ }^{5}$ or when the non-unique output arguments are of a type for which sharing is not possible (integers, enums, chars, etc.). In all these cases, it is safe to conclude that the procedure will not introduce new aliases.

2. Manual aliasing annotation for foreign code. Important parts of the Mercury standard library consist of procedures which are defined in terms of foreign code. With the intention to be used mainly in this standard library, we have extended the Mercury language such that foreign code can be manually annotated with aliasing-information.

\footnotetext{
${ }^{5}$ A procedure call cannot create additional aliases between input variables as they must be ground at the moment when the procedure is called.
} 
3. Manual iteration for mutual dependent modules. The current compilationscheme of Mercury is not yet able to cope with mutual dependent modules. Consider a module $\mathrm{A}$ in which some procedures are expressed in terms of procedures declared in a module $\mathrm{B}$, and vice versa. The normal compilation scheme is to compile one of the files, and then the other one. In the presence of an optimizing compiler this is not enough. At the moment the first module is compiled, nothing is known from the second one, yielding bad precision for the first one. This bad precision will propagate further to the second file as the second file relies on the first one. Bueno et al. [5] propose a new compilation scheme which is able to handle these cases. As this requires quite some work, we make a work around by allowing manually controlled incremental compilation.

\subsection{Making Compilation Faster: Widening the Aliasing}

While it is interesting to have more precise aliasing information than simply top, having more aliases also slows down the system. Now one can argue that speed is not a major requirement of a CTGC-system as it is primarely intended to be used only at the final compilation phase of a program, but even for our benchmarks we were not ready to wait hours for a module to compile. Therefore, in order to produce a usable CTGC-system we have added a widening operator [6] which acts upon the aliases produced ${ }^{6}$.

During the data-flow analysis, a datastructure is represented by its full path down the term it is part of. Such a path is a concatenation of selectors which selects the functor and the exact argument position in the functor ${ }^{7}$. Aliases are expressed as pairs of datastructures.

To illustrate this, let us consider the following definition of a tree type:

:- type tree ---> e ; two(int,tree,tree)

; three(int, int, tree,tree,tree).

After the construction $\mathrm{V}<=\operatorname{three}(2,3, \operatorname{two}(0, e, e), A, A)$ (where $\mathrm{A}$ is a variable bound to another tree-term), the path $($ three, 3$) \cdot($ two, 1$)$ selects in $\mathrm{V}$ the zerointeger. The path (three, 3 ) selects in $\mathrm{V}$ the whole datastructure corresponding to the first subtree (namely two $(0, e, e)$ ). In $\mathrm{V}$, the positions corresponding with the paths (three, 4$)$ and (three, 5$)$ are aliased.

For the aliasing information, we introduced type widening that consists of replacing a full path of normal selectors by one selector, a so called type selector. The meaning of a type selector is as follows: instead of selecting one specific subterm of a term, it will select all the subterms which have the type expressed by the selector. In our example, the paths (three, 1$),($ three, 2$)$, and

\footnotetext{
${ }^{6}$ This widening operator can be enabled on a per-module base. The user can also specify the threshold at which widening should be performed: e.g. only widen if the size of the set of aliases exceeds 1000 .

${ }^{7}$ Infinite paths are avoided by simplifying full type trees to type graphs. This is beyond the scope of this paper.
} 
$($ three, 3$) \cdot(t w o, 1)$ all select integer elements of $\mathrm{V}$. With type widening, all these selectors are reduced to the selector (int), i.e. the type of the subterms which they select. The alias in V (ie. between (three, 4) and (three,5)) becomes an alias between (tree) and (tree), hence expressing that all subtrees of $\mathrm{V}$ might be aliased. If other aliases between subtrees of $\mathrm{V}$ exist, then they will all be replaced by this one single alias, hence making the overall size of the set of aliases smaller.

This widening leads to a considerable speed-up of the CTGC-system (compilation of some modules taking almost one hour was now reduced to less than a minute). Our results suggest that the overall precision remains sufficient in order to detect the expected reuses for our benchmarks.

\section{$5 \quad$ First Results}

We have evaluated the effectiveness of our CTGC-system by comparing memory usage and measuring compilation times. We have used toy benchmarks and one real-life program. All the experiments were run on an Intel-Pentium III (600Mhz) with 256MB RAM, using Debian Linux 2.3.99, under a usual workload. The CTGC-system was integrated into version 0.9.1 of the MMC. The reported memory information is obtained using the MMC memory profiler. This profiler counts the total number of memory words that are allocated on the heap ${ }^{8}$. The timings are averages of 10 runs each time. All the benchmarks are compiled using a non-optimized Mercury standard library w.r.t. memory usage (hence no reuse in the library predicates ${ }^{9}$ ). This allows us to focus on the reuse occurring in the actual code of the benchmarks.

The toy benchmarks comprise nrev (naive reverse of a list of 3000 integers), qsort (quick sort of a sorted list of 10000 integers), and argo_cnters (a benchmark counting various properties of a file, also used in [15]). Table 1 shows the results. These are independent of the CTGC configuration used, as they all yield the same results here. For each of the benchmarks every possible reuse is detected, yielding the expected savings in memory usage and execution time.

\begin{tabular}{|l||c|c|c||c|c|c|c|}
\hline \multicolumn{1}{|c||}{} & \multicolumn{3}{c||}{ No Reuse } & \multicolumn{4}{c|}{ Reuse } \\
\cline { 2 - 8 } module & C (sec) & M (Word) & R (sec) & C (sec) & M (Word) & m (\%) & R (sec) \\
\hline nrev & 1.49 & $9 \mathrm{M}$ & 1.51 & 11.79 & $6 \mathrm{k}$ & -99.9 & 0.32 \\
qsort & 1.40 & $50 \mathrm{M}$ & 36.63 & 11.29 & $20 \mathrm{k}$ & -99.9 & 27.22 \\
argo_cnters & 4.53 & $3.00 \mathrm{M}$ & 0.35 & 16.38 & $2.60 \mathrm{M}$ & -13.3 & 0.32 \\
\hline
\end{tabular}

Table 1. Toy benchmarks. $\mathrm{C}=$ compilation time. $\mathrm{M}=$ number of allocated words. $\mathrm{R}$ $=$ execution time. $\mathrm{m}=$ relative reduction in memory usage.

\footnotetext{
${ }^{8}$ Note that this count is independent of any run-time garbage collection.

${ }^{9}$ Normally, a Mercury system with CTGC would also have the library modules compiled with CTGC in the same way as user modules.
} 
Next to small benchmarks, we found it important to evaluate the system on a real-life program, where the different constraints and strategies do make a difference. The program we used is a ray tracer program developed for the ICFP'2000 programming contest [17] where it ended up fourth. This program transforms a given scene description into a rendered image. It is a CPU- and memory-intensive process, and therefore an ideal candidate for our CTGC-system to be tested on. A complete description of this program can be found at [20].

The program consists of 20 modules (5700 lines of code), containing mostly deterministic predicates. All modules could be compiled without widening, except for one: peephole. This module manipulates complex constructors and generates up to $11 \mathrm{~K}$ aliases. Without type-widening, the compilation of peephole takes 160 minutes. With type-widening (at 500 aliases), it only takes 40 seconds. The compilation of the whole program with CTGC (and widening) takes 5 minutes, compared to 1 minute for a normal compilation. As some of these modules depend on each other, the technique of manually iterating the compilation was used to obtain better results. For this benchmark, the compilation had to be repeated 3 times to reach a fixpoint (for a total time of 15 minutes). Each time every module was recompiled. In a smart compilation environment, most of the recompilations could be avoided.

To measure the effects of the different constraints and strategies we have compiled the ray tracer with different CTGC-configurations. The first row of Table 2 shows the number of memory words and the execution time (in seconds) needed to render a set of 27 different scene descriptions (ranging from simple scenes, to more complex ones) using a version of the ray tracer without CTGC. Rows 1 to 9 show the relative memory usage and execution time of ray tracers compiled using different CTGC-configurations for the same set of scene descriptions:

- Using the matching arities (match) or label-preserving (same cons) constraints, up to $24 \%$ memory can be saved globally. For some scene descriptions, this can go up to $30 \%$. There is also a noticeable speedup (14\%).

- Using almost matching arities within a distance of one (within 1) or two (within 2), much less memory is saved (only 10\%) with hardly any speedup. The bad memory usage is not surprising as none of the selection strategies takes into account the correspondance of the arities between a new cell and the available dead cells. The bad timings are also explicable: with nonmatching arities, reuse leaves space-leaks which cannot immediately be detected by the current run-time garbage collector, hence the garbage collector will be called more often. Improvements to the garbage collector are required.

- Globally, using the random selection strategy yields slightly worse results than lifo. For some scene descriptions though, results are better, but without spectacular differences.

- Row 9 shows the results of a ray tracer compiled using a version of the Mercury standard library with CTGC. There is hardly any difference with Row 1, where libraries were used without CTGC. This is due to the fact that the ray tracer makes a limited use of these libraries. 


\begin{tabular}{|l|c|c|c||c|c|c|c|}
\hline & \multicolumn{3}{|c||}{ Configuration } & \multicolumn{2}{c|}{ Memory } & \multicolumn{2}{c|}{ Time } \\
& \multicolumn{2}{|c|}{} & \multicolumn{2}{|c|}{ (kWord) } & \multicolumn{2}{c|}{$(\%)$} & \multicolumn{2}{c|}{$(\mathrm{sec})$} & $(\%)$ \\
\hline 0 & \multicolumn{2}{|c|}{ no CTGC } & & 1024795.51 & - & 362.31 & - \\
\hline 1 & lifo & match & & 776707.92 & -24.21 & 311.85 & -13.93 \\
2 & lifo & same cons & & 791742.06 & -22.74 & 313.57 & -13.45 \\
3 & lifo & within 1 & & 916642.90 & -10.55 & 361.84 & -0.13 \\
4 & lifo & within 2 & & 917847.97 & -10.44 & 359.90 & -0.67 \\
5 & random & match & & 780838.58 & -23.81 & 310.75 & -14.23 \\
6 & random & same cons & & 795872.67 & -22.34 & 312.70 & -13.69 \\
7 & random & within 1 & & 920764.26 & -10.15 & 359.14 & -0.87 \\
8 & random & within 2 & & 921969.35 & -10.03 & 355.08 & -2.00 \\
9 & lifo & match & libs & 775607.04 & -24.32 & 320.32 & -11.59 \\
\hline 10 & lifo & match & cc & 513901.37 & $-\mathbf{4 9 . 8 5}$ & 301.66 & -16.74 \\
11 & lifo & same cons & cc & 542626.80 & $\mathbf{- 4 7 . 0 5}$ & 304.20 & -16.04 \\
12 & lifo & within 1 & cc & 845603.55 & -17.49 & 375.79 & 3.72 \\
13 & lifo & within 2 & cc & 864722.90 & $\mathbf{- 1 5 . 6 2}$ & 370.49 & 2.26 \\
14 & random & match & cc & 518032.04 & $\mathbf{- 4 9 . 4 5}$ & 299.45 & -17.35 \\
15 & random & same cons & cc & 546757.48 & $\mathbf{- 4 6 . 6 5}$ & 302.79 & -16.43 \\
16 & random & within 1 & cc & 849724.90 & -17.08 & 363.90 & 0.44 \\
17 & random & within 2 & cc & 868844.29 & $\mathbf{- 1 5 . 2 2}$ & 391.68 & 8.11 \\
\hline
\end{tabular}

Table 2. ICFP-ray tracer using different CTGC-configurations.

Finally, a version of the ray tracer was built without type-widening (lifo and matching arities). Compared to row 1 in Table 2 the overall memory usage difference is less than $1 \%$. The execution times are comparable.

\section{Non-local Reuse: Cell Cache}

Currently we have assumed that all dying datastructures must be reused locally, i.e. within the same procedure in which they die. Hence quite some interesting possibilities of reuse could be missed.

We see three ways to achieve non-local reuses as well. The first and the most difficult is to extend the data-flow analysis to handle non-local reuse. The analysis would have to propagate possible dead cells and thus become quite complex. It would also require intensive changes in the internal calling convention of procedures within the MMC as the address of the cells to be reused would have to be passed between procedures. The second approach is to combine reuse analysis with inlining in such a way that the cell death and subsequent reuse end up in the same procedure. The third approach, which is the one we implemented, is to cache dead cells. Whenever a cell dies unconditionally and cannot be reused locally, we mark it as cacheable. At runtime the address of the cell as well as its size will be recorded in a cache (or free list). Before each memory allocation the runtime system will first check the cell cache to see if a cell of the correct 
size is available and use that cell instead of allocating a new cell. This operation increases the time taken to allocate a memory cell in the case of the cell cache being empty, and hence should only be a win if the cell cache occupancy rate is high. It also avoids new allocations so the overall cost of the runtime garbage collection system should go down due to smaller heap sizes and less frequent need for garbage collection.

The $c c$-entries of Table 2 (Rows 10-17) show the results of CTGC-configurations combined with the cell cache technique. Compared to the basic CTGCconfigurations, cell caching always increases memory savings, going up to $49 \%$ (for some scenes even 70\%). In the case of label-preserving or matching arities constraints, execution time drops slightly. On the other hand, using almost matching arities combined with cell caching increases the execution time.

\section{$7 \quad$ Further Improvements}

In the near future, we intend to explore a number of improvements to our system. First, for some procedures, several possibilities of reuse are discovered, each one imposing its own reuse conditions. Taken together, these reuse conditions are too restrictive on the caller, hence hardly any calling environment is able to satisfy them, and no reuse is performed at all. A top-down call-dependent version splitting pass could aid in generating more useful reuse-versions of procedures, and avoid the generation of the useless ones.

A second problem is the too absorbant effect of the notion of top currently used in the alias information. Once top is encountered, it propagates all throughout the remainder of the code. Instead of top, we could use topmost substitutions [4]: e.g. generating all possible combinations of aliases between the arguments of a called predicate, based on the types of these arguments, either explicitly or in a more compact form (using type-selectors or keeping sets of variables, stating that these variables might be aliased to each other in any possible way).

In this paper we mainly focussed on memory savings, reasoning that saving memory implies less garbage collection, hence diminishes the execution time. If execution time is of primary concern, than more sophisticated reuse strategies will be needed. In the near future we will adopt the use of weighted graphs [7] where the weights can be adjusted for minimizing memory usage or execution time (taking into account the fields that do not need to be updated). We will also consider splitting dead cells and reusing them for different new cells.

In [10] the focus on execution time is even greater, trying to discover almost every field not requiring an update, going even beyond the boundaries of single procedures. This is indeed important in Prolog, where the determinism of procedures is not necessarely known at analysis time, and where given the underlying data-flow analysis, each cell update requires extra care in the case the value has to be reset upon backtracking. In Mercury, where determinism is known at compilation time, and where the analysis explicitly takes into account backtracking, this is not a major issue. Therefore, it is not our immediate intention to try to avoid every possible cell update. 


\section{Conclusion}

This paper describes a complete working compile-time garbage collection system for Mercury, a logic programming language with declarations. The system consists of three passes: data-flow analysis, reuse decision, and low level code generation. The data-flow analysis based on [15] detects which cells become available for reuse. This paper presents easy implementable restrictions, constraints and strategies for selecting realistic reuses. In order to obtain a workable CTGCsystem, low level improvements were introduced.

A major contribution of this work is the integration of the CTGC system in the Melbourne Mercury Compiler and its evaluation. Some small benchmarks were used, but also one real-life complex program, a ray tracer. Average global memory savings of up to $49 \%$ were obtained, with a speedup of up to $17 \%$. It would be interesting to compare these results with the total potential of reuse within the program. This total potential could be approximated using the techniques used in our first prototype [15] to predict the amount of reuse.

Beside the proposed improvements the system could also be adapted to handle higher order calls and type classes properly (instead of generating top aliasing, and not allowing reuse). Yet given the fact that many higher order calls are specialized away by the compiler, we currently do not believe that the overhead needed to deal with these language constructs is worthwhile.

\section{References}

1. Y. Bekkers and P. Tarau. Monadic constructs for logic programming. In J. Lloyd, editor, Proceedings of the International Symposium on Logic Programming, pages 51-65, Cambridge, Dec. 4-7 1995. MIT Press.

2. M. Bruynooghe. A practical framework for the abstract interpretation of logic programs. Journal of Logic Programming, 10(2):91-124, Feb. 1991.

3. M. Bruynooghe, G. Janssens, and A. Kågedal. Live-structure analysis for logic programming languages with declarations. In L. Naish, editor, Proceedings of the Fourteenth International Conference on Logic Programming (ICLP'g7), pages 3347, Leuven, Belgium, 1997. MIT Press.

4. F. Bueno, D. Cabeza, M. Hermenegildo, and G. Puebla. Global Analysis of Standard Prolog Programs. In European Symposium on Programming, number 1058 in LNCS, pages 108-124, Sweden, April 1996. Springer-Verlag.

5. F. Bueno, M. García de la Banda, M. Hermenegildo, K. Marriott, G. Puebla, and P. J. Stuckey. A model for inter-module analysis and optimizing compilation. In Tenth International Workshop on Logic-based Program Synthesis and Transformation, London, UK, 2000. to appear.

6. P. Cousot and R. Cousot. Comparing the Galois connection and widening/narrowing approaches to abstract interpretation. In M. Bruynooghe and M. Wirsing, editors, Proceedings of the Fourth International Symposium on Programming Language Implementation and Logic Programming, pages 269-295, Leuven, Belgium, 1992. LNCS 631, Springer-Verlag.

7. S. K. Debray. On copy avoidance in single assignment languages. In D. S. Warren, editor, Proceedings of the Tenth International Conference on Logic Programming, pages 393-407, Budapest, Hungary, 1993. The MIT Press. 
8. B. Demoen, M. García de la Banda, W. Harvey, K. Marriott, and P. J. Stuckey. An overview of HAL. In Proceedings of the International Conference on Principles and Practice of Constraint Programming, pages 174-188, Virginia, USA, October 1999. Springer Verlag.

9. T. Dowd, Z. Somogyi, F. Henderson, T. Conway, and D. Jeffery. Run Time Type Information in Mercury. In Principles and Practice of Declarative Programming, pages 224-243, 1999.

10. G. Gudjónsson and W. H. Winsborough. Compile-time memory reuse in logic programming languages through update in place. ACM Transactions on Programming Languages and Systems, 21(3):430-501, May 1999.

11. F. Henderson, T. Conway, Z. Somogyi, and D. Jeffery. The Mercury language reference manual. Technical Report 96/10, Dept. of Computer Science, University of Melbourne, February 1996.

12. M. Hermenegildo, F. Bueno, G. Puebla, and P. López. Program Analysis, Debugging and Optimization Using the Ciao System Preprocessor. In D. D. Schreye, editor, 1999 International Conference on Logic Programming, pages 52-66, Cambridge, MA, December 1999. MIT Press.

13. A. Kågedal and S. Debray. A practical approach to structure reuse of arrays in single assignment languages. In L. Naish, editor, Proceedings of the 14 th International Conference on Logic Programming, pages 18-32, Cambridge, July 8-11 1997. MIT Press.

14. F. Kluźniak. Compile-time garbage collection for ground Prolog. In R. A. Kowalski and K. A. Bowen, editors, Proceedings of the Fifth International Conference and Symposium on Logic Programming, pages 1490-1505, Seattle, 1988. MIT Press, Cambridge.

15. N. Mazur, G. Janssens, and M. Bruynooghe. A module based analysis for memory reuse in Mercury. In J. Lloyd, V. Dahl, U. Furbach, M. Kerber, K.-K. Lau, C. Palamidessi, L. Moniz Pereira, Y. Sagiv, and P. J. Stuckey, editors, Computational Logic - CL 2000, First International Conference, London, UK, July 2000, Proceedings, volume 1861 of Lecture Notes in Artificial Intelligence, pages 1255-1269. Springer-Verlag, 2000.

16. M. Mohnen. Optimising the Memory Management of Higher-Order Functional Programs. Technical Report AIB-97-13, RWTH Aachen, 1997. PhD Thesis.

17. G. Morrisett and J. Reppy. The third annual ICFP programming contest. In Conjunction with the 2000 International Conference on Functional Programming, http://www.cs.cornell.edu/icfp/, 2000.

18. A. Mulkers, W. Winsborough, and M. Bruynooghe. Live-structure dataflow analysis for Prolog. ACM Transactions on Programming Languages and Systems, 16(2):205-258, Mar. 1994.

19. Z. Somogyi, F. Henderson, and T. Conway. The execution algorithm of Mercury, an efficient purely declarative logic programming language. The Journal of Logic Programming, 29(1-3):17-64, October-December 1996.

20. The Mercury Team. ICFP 2000: The merry mercurians. Description of the Mercury entry to the ICFP'2000 programming contest, http://www.mercury.cs.mu.oz.au/information/events/icfp2000.html.

21. M. Tofte and J.-P. Talpin. Region-based memory management. Information and Computation, 132(2):109-176, 1997.

22. P. Wadler. The essence of functional programming. In Conference Record of the Nineteenth Annual ACM SIGPLAN-SIGACT Symposium on Principles of Programming Languages, pages 1-14, Albequerque, New Mexico, Jan. 1992. 